\title{
RED VARIABLE STARS IN METAL RICH GLOBULAR CLUSTERS
}

\author{
T. LLOYD EVANS \\ Radcliffe Observatory, Pretoria; Royal Observatory, Edinburgh \\ and \\ J. W. MENZIES \\ Radcliffe Observatory, Pretoria; University Observatory, Oxford
}

\section{Introduction}

The globular clusters contain sufficiently large numbers of stars to permit a systematic study of the intrinsically rare variable stars which lie near the tip of the red giant branch. The position of the smaller amplitude stars in the colour magnitude diagram is of particular interest. Eggen (1972) has published photoelectric observations of such stars in several globular clusters, most of intermediate or low metal abundance. The mean colour of 14 stars in 5 clusters is $\left(V-I_{K}\right)=1.40$, with a spread from 1.60 to 1.12 (or 0.75 if V8 in M22 is of this type) which Eggen regards as indicating a range of temperature. The red variable stars in the metal rich globular cluster 47 Tucanae are much redder and show a considerable range of colour.

It is of interest to know whether the colours of the red variable stars in globular clusters depend on the metal content of the cluster. The colour magnitude diagrams on the $V, I_{K}$ system have been found for a sample of metal-rich clusters containing red variable stars, many of which are new discoveries.

$I_{K}$ is the infrared magnitude on the system of Kron and Smith (1951). The clusters were selected for a small (negative) value of $Q$ (van den Bergh, 1967), late spectral type, and weak or nonexistent blue horizontal branch, as well as observational convenience and the availability of supporting data. Details of the observations, made with the $1.88 \mathrm{~m}$ reflector at Pretoria, will be published elsewhere.

\section{The Variable Stars}

The available plates have been searched for variable stars. The number of plates, most of which are on the $V$ system, is quite small and the search cannot be considered exhaustive. The high proportion of variables near the red giant tip shows that in certain clusters a substantial proportion of such variables have been detected, however. Some variables will have escaped detection because they were always at the same phase, always below the plate limit, or because they happened not to vary much over the period of the observations; another major problem in detection is that caused by the very crowded fields involved.

All suitable plate pairs were blinked at the Radcliffe Observatory or at the University of St. Andrews Observatory. Many of these plates, and others which were not taken specifically for the study of variable stars, were measured with iris photometers. 
TABLE I

Globular clusters

\begin{tabular}{rrllll}
\hline NGC & Epochs & Seasons & $E(B-V)$ & $E(V-I)$ & $Q$ \\
\hline 104 & 5 & 2 & 0.07 & 0.08 & -0.26 \\
5927 & 11 & 4 & 0.60 & 0.72 & -0.13 \\
6171 & 2 & 1 & 0.28 & 0.35 & -0.35 \\
6352 & 8 & 4 & 0.30 & 0.36 & -0.09 \\
6356 & 3 & 1 & 0.39 & 0.47 & -0.24 \\
6388 & 3 & 2 & 0.36 & - & -0.20 \\
6553 & 23 & 6 & 0.71 & - & -0.11 \\
6637 & 11 & 3 & 0.20 & - & -0.21 \\
6712 & 2 & 2 & 0.48 & 0.58 & -0.31 \\
6723 & Many & 6 & 0.00 & 0.00 & -0.28 \\
\hline
\end{tabular}

TABLE II

Variable stars

\begin{tabular}{|c|c|c|c|c|c|}
\hline Cluster & Star & $\Delta V$ & $A_{V}$ & $\left(V-I_{K}\right)$ & Remarks \\
\hline \multirow[t]{24}{*}{104} & V1 & $3+$ & 4.4 & - & $\operatorname{Mira} P=212^{\mathrm{d}}$ \\
\hline & $\mathrm{V} 2$ & $3+$ & 3.7 & - & Mira $P=203^{d}$ \\
\hline & V3 & $5:$ & 4.3 & - & $\operatorname{Mira} P=192^{\mathrm{d}}$ \\
\hline & V4 & 1.2 & 1.8 & +3.16 & $P=165^{\mathrm{d}}$ \\
\hline & V5 & 0.3 & 0.4 & +1.92 & M2III: $P=45^{\mathrm{d}}$ \\
\hline & V6 & 0.2 & 0.6 & +1.87 & $P=47^{\mathrm{d}}$ \\
\hline & V7 & 0.5 & 0.5 & +2.34 & M2: $\quad P=58^{\mathrm{d}}$ \\
\hline & V8 & 0.7 & 0.7 & +2.46 & M2-3II: $P=150^{d}$ \\
\hline & V11 & 0.6 & $0.8\left(\mathrm{~m}_{\mathrm{pg}}\right)$ & +2.69 & HV813 W12 \\
\hline & V13 & 0.5 & - & +1.83 & Wilkens $^{\mathrm{a}}$ W173 \\
\hline & W300 & 0.3 & - & +2.09 & Arp et al. (1963) \\
\hline & R18 & 0.2 & - & +1.94 & M Brooke (1969) \\
\hline & W81 & 0.1 & - & +2.00 & Eggen (1972) \\
\hline & L168 & 0.3 & & +2.13 & b \\
\hline & R10 & 0.6 & & $+1.60:$ & K311 \\
\hline & A1 & 0.8 & & +2.26 & \\
\hline & A2 & 1.0 & & $+2.86:$ & \\
\hline & A4 & 0.5 & & +2.05 & \\
\hline & A6 & 0.6 & & +1.82 & \\
\hline & A8 & 0.3 & & +1.78 & \\
\hline & A9 & 0.5 & & +2.13 & \\
\hline & $\mathrm{A} 13$ & - & & +2.48 & Crowded \\
\hline & A18 & 0.3 & & +2.48 & \\
\hline & LR5 & 0.4 & & +2.31 & \\
\hline \multirow[t]{10}{*}{5927} & FL4 & 0.6 & & +3.93 & Non-member? \\
\hline & FL14 & 0.6 & & $+3.13:$ & Crowded \\
\hline & $\mathrm{V} 1$ & $3+$ & & - & Mira $P \sim 300^{\mathrm{d}}$ \\
\hline & V3 & 0.7 & & +3.40 & \\
\hline & V6 & 0.5 & & +3.44 & \\
\hline & V7 & 0.6 & & +3.05 & \\
\hline & V8 & 0.6 & & +3.37 & \\
\hline & V9 & 0.6 & & +3.64 & \\
\hline & V10 & 0.9 & & +3.69 & \\
\hline & L43 & 0.4 & & +3.11 & \\
\hline
\end{tabular}


Table II (Continued)

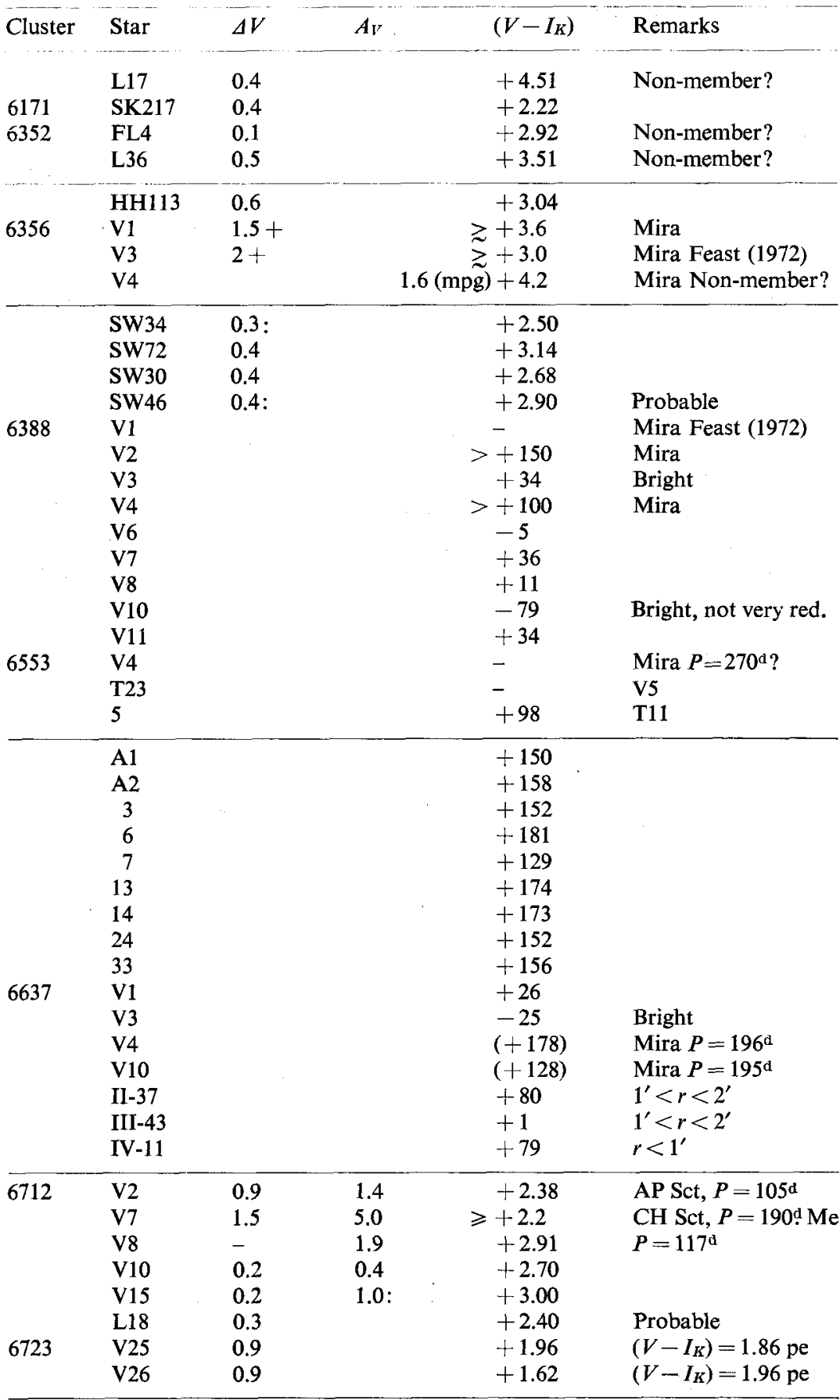

a See Fourcade and Laborde (1966).

b Marked VII on finding chart, in error. 


\section{Notes to Table II}

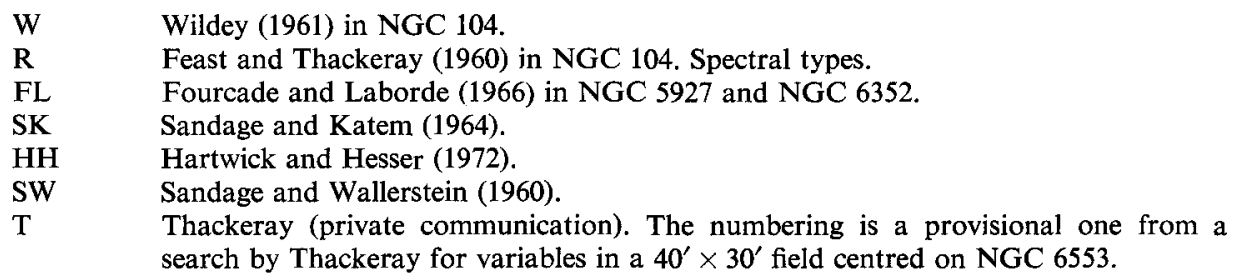

NGC 6637: see Hartwick and Sandage (1968), Rosino (1962), Catchpole et al. (1970), Lloyd Evans and Menzies (1971).

VI in NGC 5927 is Osborne's (1968) variable; otherwise stars identified by $V$, above a ruled line, are numbered according to Hogg (1955). Identifications below the line are assigned by the authors.

The values given for $\left(V-I_{K}\right)$ for NGC 6388,6553 and 6637 are the mean difference of iris readings, $S_{I}-S_{V}$.

Stars denoted as non-members are considered to lie outside the cluster.

Criteria for deciding on the reality of variability included agreement of blink microscope and iris photometer results and confirmation from additional plates taken on the same nights. Judgement had to be exercised especially where the star concerned lay near the centre of the cluster. Magnitude differences of 0.2 might be considered real in favourable cases but the detection threshold was larger in such compact clusters as NGC 6356 and 6388.

Table I lists for each cluster the number of epochs (dark of moon periods) for which observations are available; number of observing seasons; reddening adopted in plotting the CM diagram; and $Q$ (van den Bergh, 1967). Table II contains the star identification, from published lists where available (those above a horizontal line being already identified in the literature); light range $\Delta V$; amplitude $A_{V}$ if already known from an adequate series of observations; $\left(V-I_{K}\right)$; remarks.

Finding charts are given for NGC 6388, 6553 and the central region of NGC 104.

TABLE III

The variable stars in metal rich clusters

\begin{tabular}{llllccl}
\hline NGC & $Q$ & Sp & CM & RR & L + SR & M \\
\hline 6352 & -0.09 & - & $R$ & - & 1 & 0 \\
6553 & -0.11 & - & $R$ & 1 & 11 & 1 \\
5927 & -0.13 & G2 & $R$ & $0 ?$ & 10 & 1 \\
6388 & -0.20 & G3 & $R$ & - & 6 & 3 \\
6637 & -0.21 & G5 & $R$ & $0 ?$ & 5 & 2 \\
6356 & -0.24 & G5 & $R$ & $0 ?$ & 4 & $2(+1)$ \\
104 & -0.26 & G3 & $R$ & 3 & 21 & 3 \\
6723 & -0.28 & G2 & $B \gtrsim R$ & 27 & 2 & 0 \\
6712 & -0.31 & G4 & $R>B$ & 8 & 5 & 1 \\
6171 & -0.35 & G0-1 & $R>B$ & 21 & 1 & 0 \\
\hline 10 & & & & 52 & 66 & 14 \\
\hline
\end{tabular}

$R:$ Red stub horizontal branch

$B, R:$ relative strengths of blue and red horizontal branches

RR, L + SR, M: numbers of RR Lyrae, irregular and semi-regular, and Mira variables, respectively. 

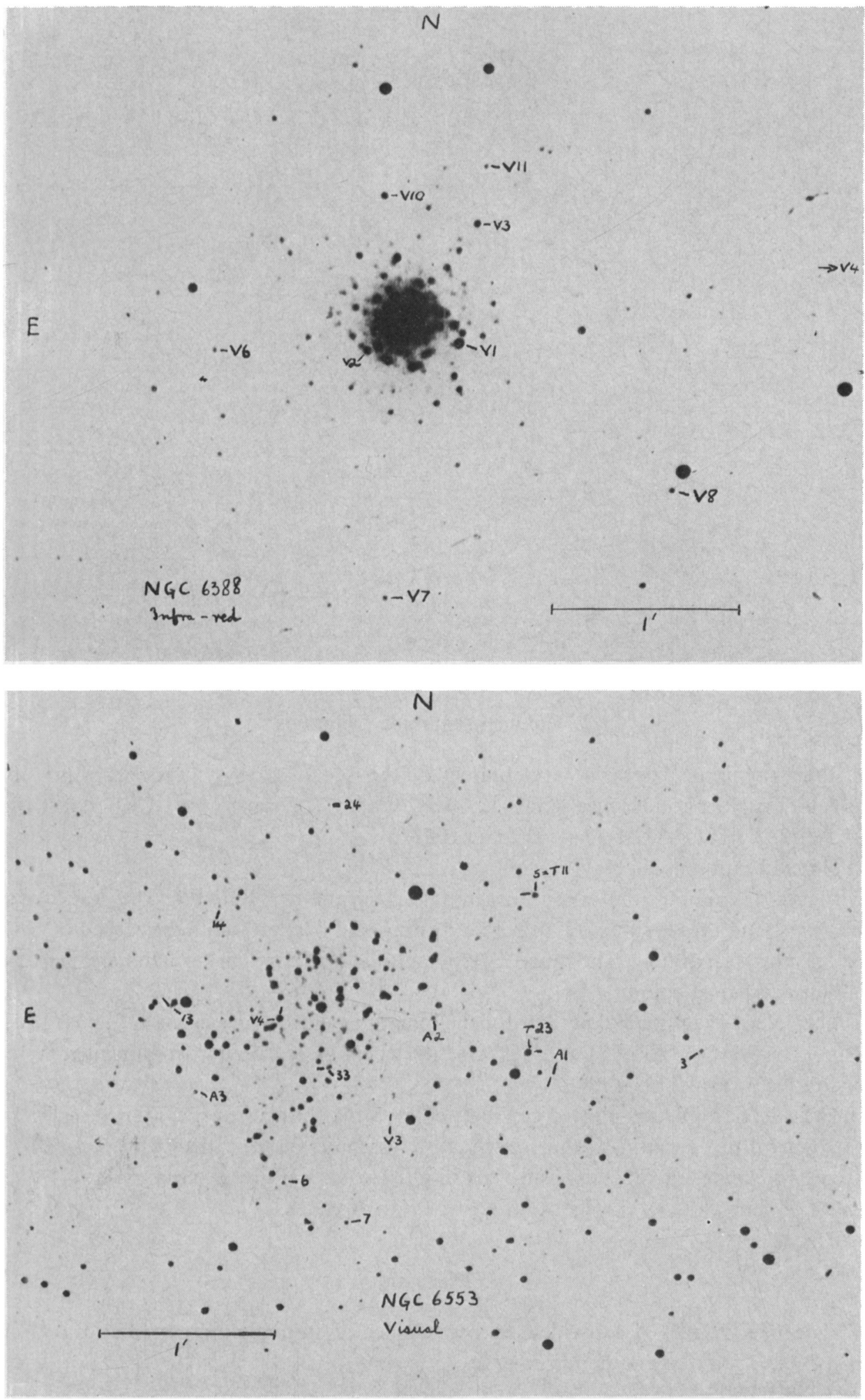


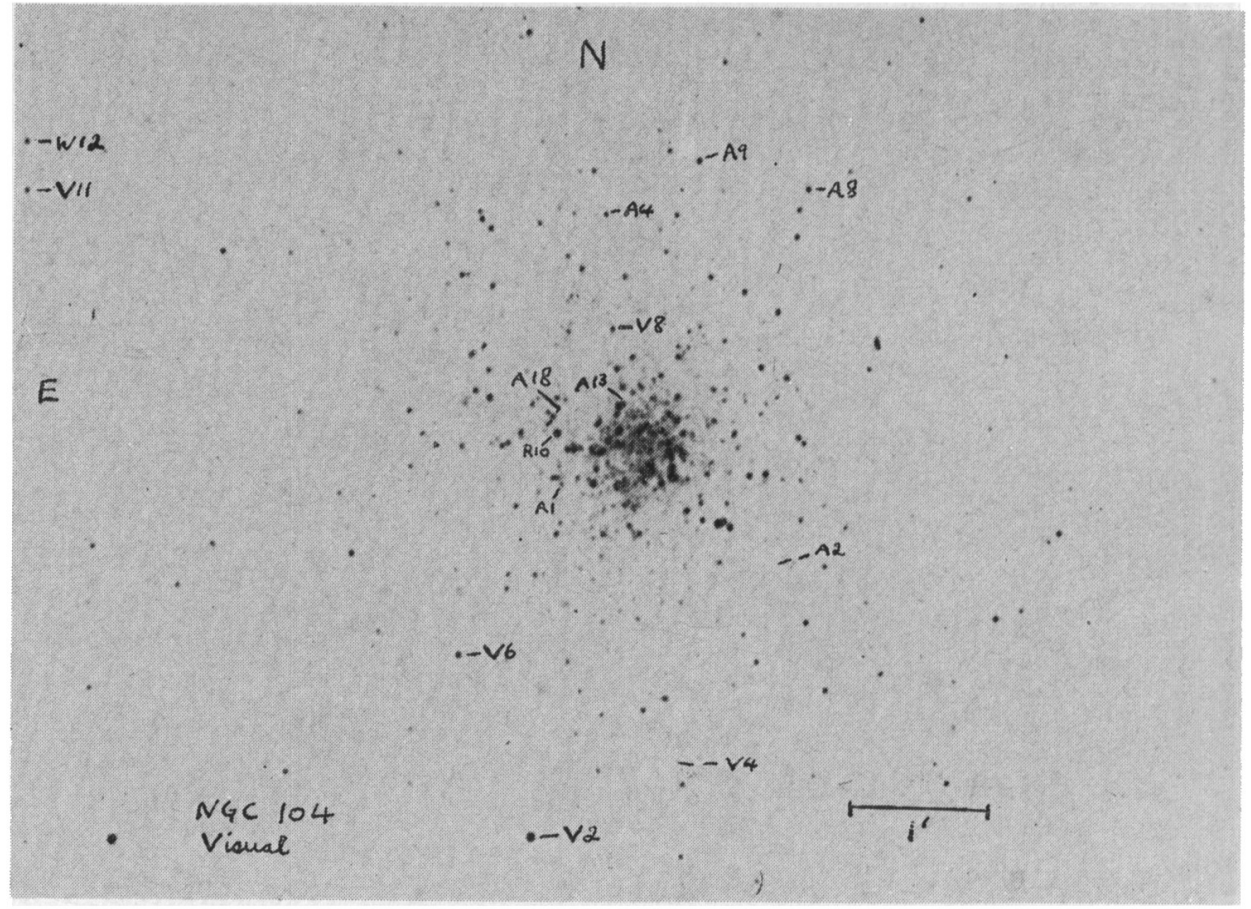

\section{Colour Magnitude Diagrams}

Colour magnitude diagrams were obtained on the $I_{K},\left(V-I_{K}\right)$ system with calibrations as follows: $V, I$ photoelectric sequence: NGC 104, 6723. Transformed $U B V$ sequence: NGC 5927, 6171, 6352, 6356, 6712 and 6723.

No calibration available: NGC 6388, 6553, 6637.

Figure 1 contains the colour magnitude diagram of NGC 104, the stars with $2^{\prime}<r<8^{\prime}$ and those with $r<2^{\prime}$ (where crowding and background light caused errors) being shown separately. The range of $V$ magnitude measured on (usually) eight plates is plotted against colour.

The colour-magnitude diagrams for the remaining clusters are given in Figures 2-5. Those for NGC 6388, 6553 and 6637 are the uncalibrated pseudo-colour-magnitude diagrams, $S_{I}$ against $S_{I}-S_{V}$.

The selection of stars plotted generally follows that of previous workers, especially with regard to the radius within which cluster members were expected to occur. The use of $V, I$ plates of short exposure has in a few cases permitted measurements to be made of stars close to the centre or crowded on $B$ plates.

\section{Discussion}

47 Tucanae (NGC 104) provides the best chance of studying the systematics of red variables. The following points emerge. 


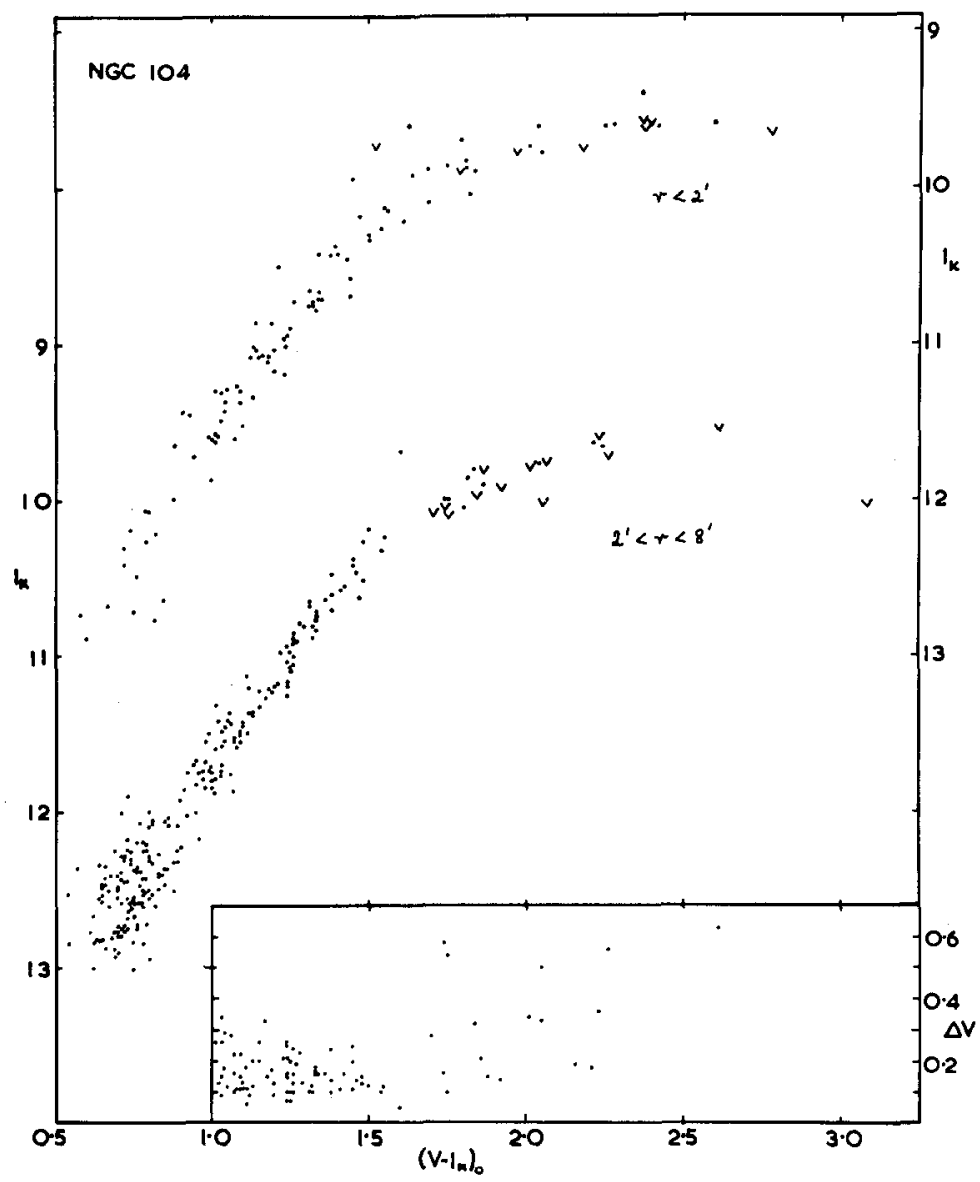

Fig. 1. Top: Stars within $2^{\prime}$ of the centre of NGC 104. All stars with $\left(V-I_{K}\right)_{0}>1.50$ are plotted but only uncrowded stars with smaller $\left(V-I_{K}\right) . I$ is uncorrected for reddening. Middle: All uncrowded stars with $2^{\prime}<r<8^{\prime}$. In both plots all stars considered variable by others are plotted as $V$, but only new cases considered definitely variable $(\Delta V \gtrsim 0 \mathrm{~m})$ ) are so indicated though other stars are suspected to vary. Lower: The range $\Delta V$ of $V$ magnitudes found on 8 plates for stars with $\left(V-I_{K}\right)_{0} \geqslant+1.00$ from the middle plot.

(1) Variability sets in at $\left(V-I_{K}\right)=1.7$.

(2) The proportion of stars which are variable, and the amplitude of variation, both increase with colour. The plot of light range against colour suggests that all stars with $\left(V-I_{K}\right)>1.8$ may be variables of small amplitude.

(3) There may be a gap, or interval of low star density, from $\left(V-I_{K}\right)=1.5$ to 1.7.

(4) The colours of stars of known spectral type (Feast and Thackeray 1960) indicate that $\left(V-I_{K}\right)=1.7$ corresponds to spectral type $\mathrm{M} 0$.

(5) The apparent downward trend in $I_{K}$ of the red giant tip may be spurious, as the average magnitude and colours plotted represent the mean of isolated measurements of magnitude instead of the average intensity which would be preferable. 


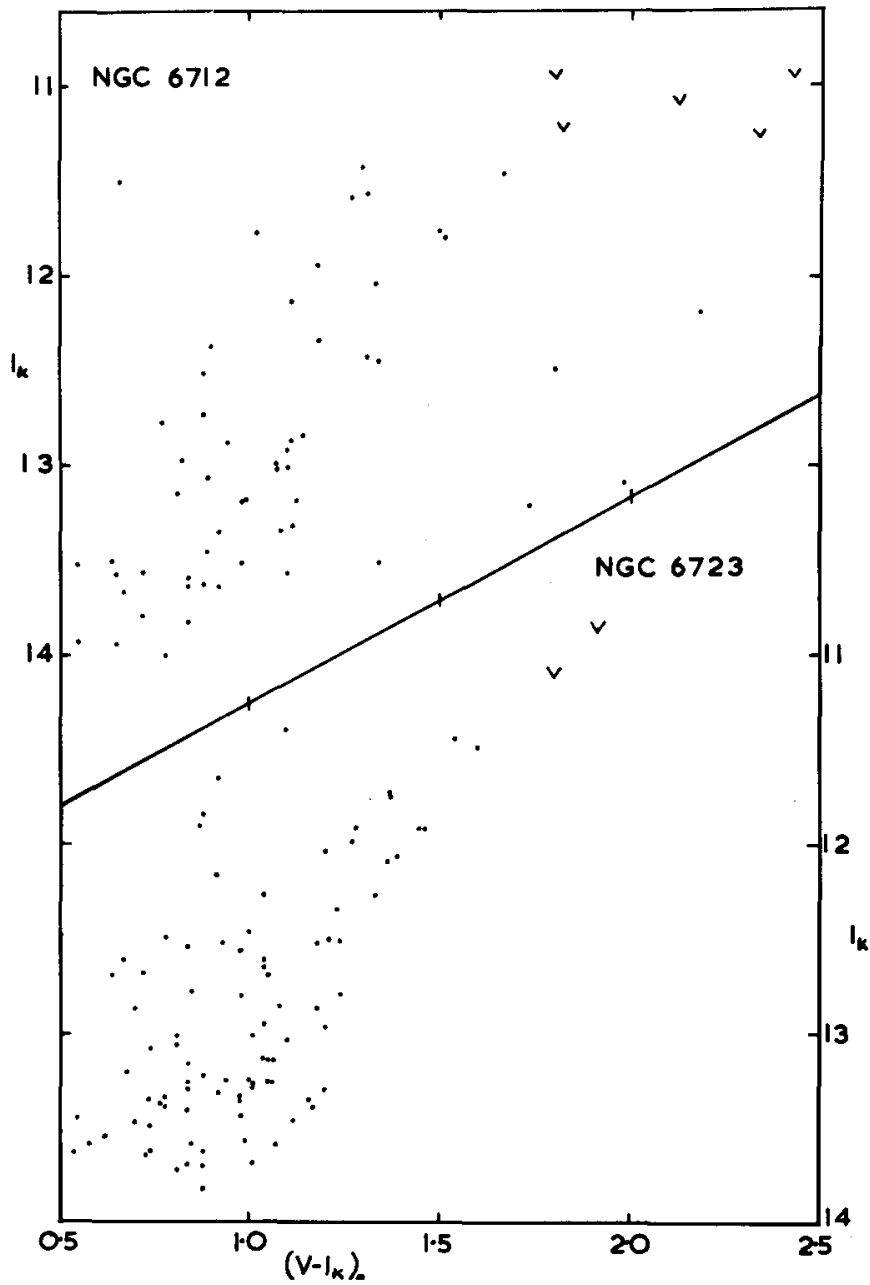

Fig. 2. Top: Stars in the region of NGC 6712, mainly those observed by Sandage and Smith (1966). Below: Stars within $4^{\prime}$ of the centre of NGC 6723. $\left(V-I_{\mathrm{K}}\right)$ but not $I$ corrected for reddening.

(6) There is no obvious clumping of variables in colour along the giant branch to parallel the clumping in period of variability reported by Arp et al. (1963).

The remaining clusters contain fewer measurable stars than NGC 104 and are perhaps best considered in groups suggested by the original selection criteria:

(A) $-0.35 \leqslant Q \leqslant-0.28$. Some blue horizontal branch and RR Lyrae stars. NGC $6171,6712,6723$.

(B) $-0.26 \leqslant Q \leqslant-0.20$. Red horizontal branch, very few RR Lyrae stars. NGC $104,6356,6388,6637$.

(C) $-0.13 \leqslant Q \leqslant-0.09$. Red horizontal branch, very few RR Lyrae stars. NGC $5927,6352,6553$.

These groups show the following properties. 
(A) The amplitudes of variability are quite large even near $\left(V-I_{K}\right)=1.8$, where variable stars are first found. The clusters are too sparse to permit a decision as to the existence of a gap to the blue of $\left(V-I_{K}\right)=1.8$. The reddest variable has $\left(V-I_{K}\right) \sim 2.4$, which is uncertain inasmuch as NGC 6712 lies in a very crowded field where cluster membership requires confirmation from radial velocities.

(B) NGC 6356, where only the tip of the giant branch has been observed, shows variables between $\left(V-I_{K}\right)=2.0$ and 2.9 , intermingled with 'non-variable' stars as in the case of NGC 104. NGC 6388 and NGC 6637, however, seem to show a sudden onset of variability with most of the stars beyond the limiting colour detected as variable on a moderate number of plates. Both these clusters as well as 47 Tuc contain variables (R10 in 47 Tuc, V3 in NGC 6637, V3 and V10 in NGC 6388) which appear brighter and in some cases bluer than the remaining variables. The spectral type of Radcliffe 10 in 47 Tuc (Feast and Thackeray 1960) is K3II, in agreement with the blue colour of $\left(V-I_{K}\right)=1.60$. The explanation for these stars may be in duplicity or in their being observed, by chance or perhaps as a result of a long wave in the light curve,

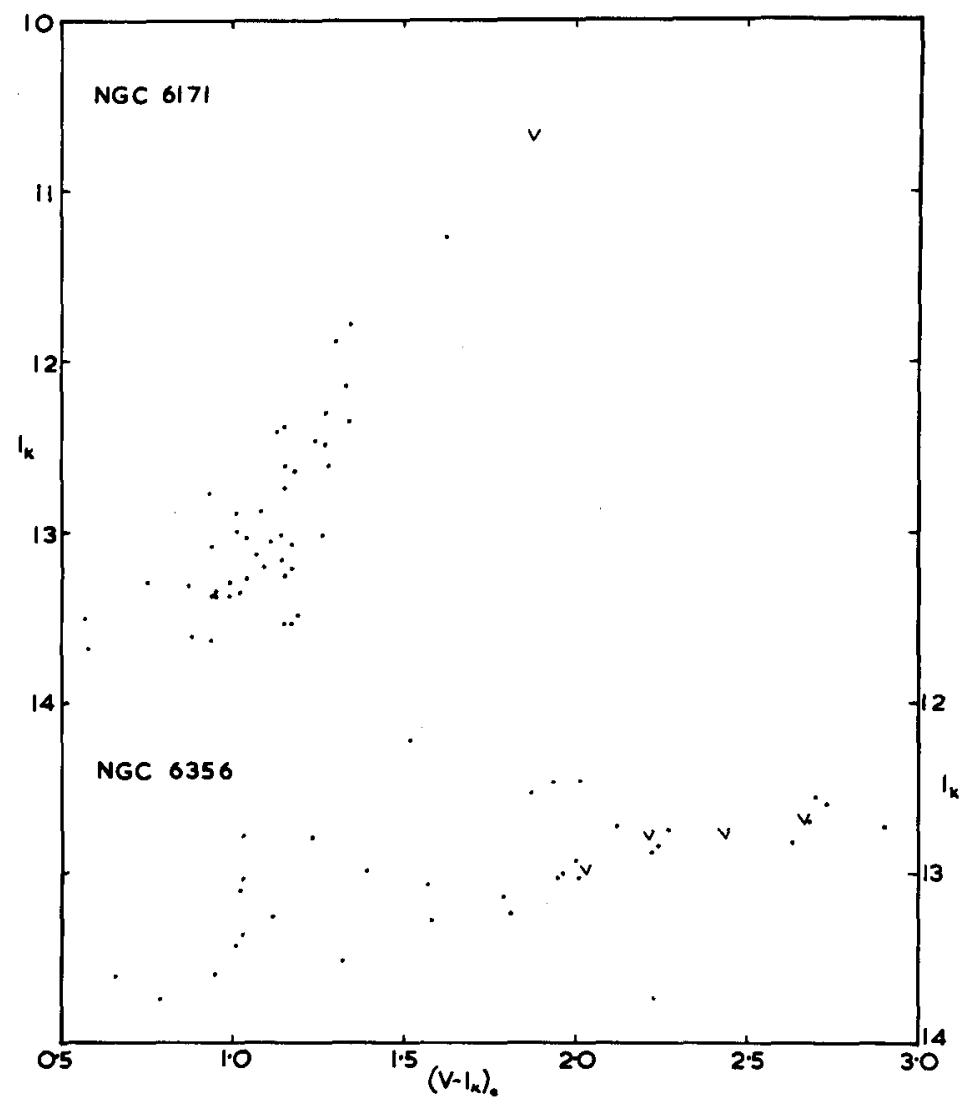

Fig. 3. Top: Stars in NGC 6171, mostly selected from those observed by Sandage and Katem (1964). Below: Stars in NGC 6356, mostly selected from those observed by Sandage and Wallerstein (1960). $\left(V-I_{K}\right)$ but not $I$ has been corrected for reddening. 
always near maximum; the few other photographs or magnitudes available from earlier work on 47 Tuc and NGC 6637 show the stars always bright. Other possible explanations are that they are at a brighter (and later?) evolutionary stage where instability sets in at a higher temperature or, speculatively, an enhanced $C / O$ ratio prevents the formation of TiO despite a low temperature. Eggen (1972) found a few similarly displaced stars in metal poor clusters, for which the last suggestion seems inappropriate.

(C) NGC 5927 contains variables of considerable amplitude with $2.3<\left(V-I_{K}\right)<3.0$. The absence of bluer variables reflects the lack of stars with $1.9<\left(V-I_{K}\right)<2.3$; the reality of either effect is uncertain, but the distribution of stars with colour is clearly different from that in 47 Tuc, and the slope of the giant branch is less. NGC 6553 seems very similar, but NGC 6352, though too poor to contribute much to the discussion, has a giant branch whose slope seems rather greater.

The main conclusion from consideration of the different groups is that the variables

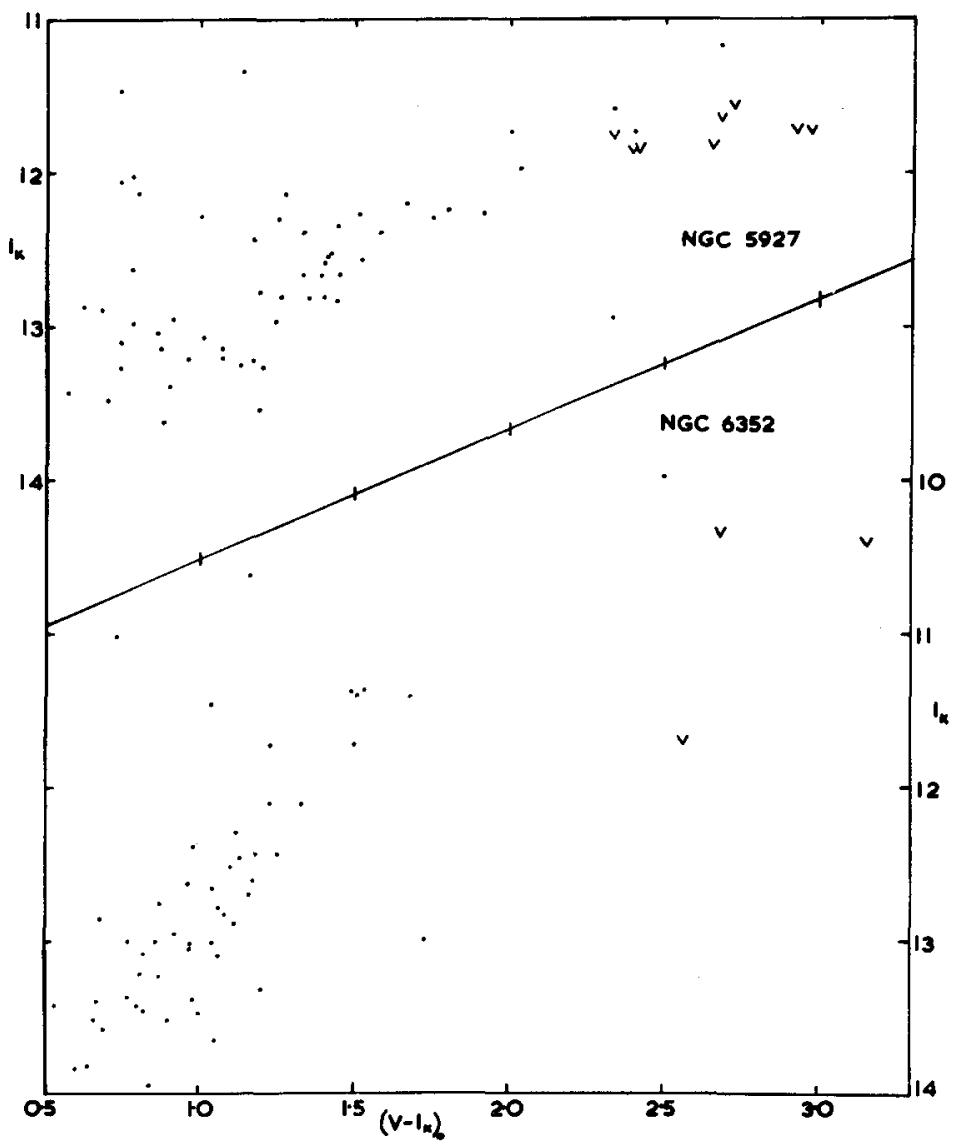

Fig. 4. Top: Stars within $2^{\prime}$ of the centre of NGC 5927. Below: Stars within $4^{\prime}$ of the centre of NGC 6352. $\left(V-I_{K}\right)$ but not $I$ has been corrected for reddening. 


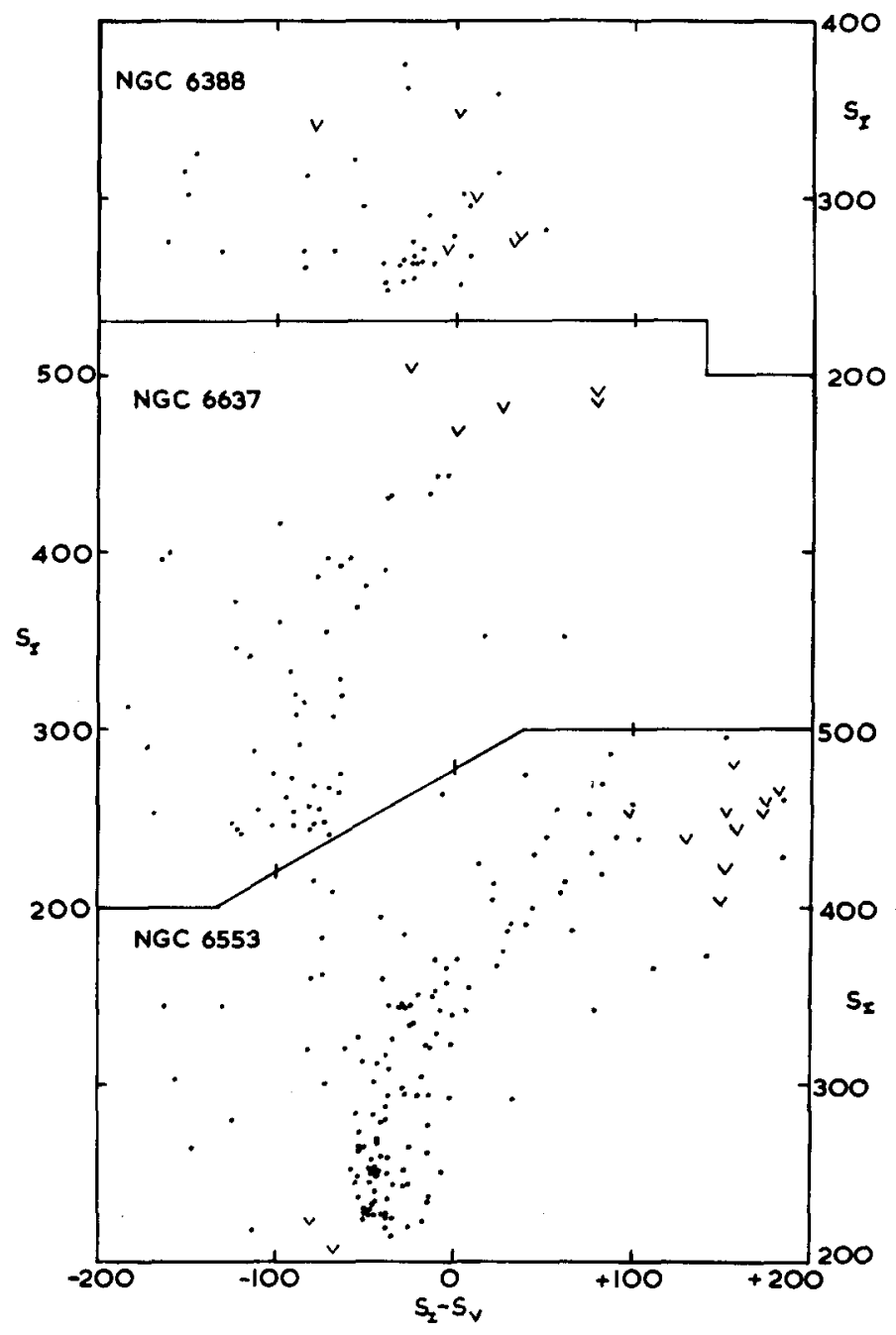

Fig. 5. Pseudo-colour-magnitude diagrams for Top: Stars within 3:3 of the centre of NGC 6388 . Middle: Stars within $2^{\prime}$ of the centre of NGC 6637. Below: Stars within 2:2 of the centre of NGC 6553 .

are redder on average as one goes from the metal-poor clusters to $\mathrm{A}$ and from $\mathrm{A}$ to $\mathrm{C}$, which is generally thought to continue in the direction of increasing metal abundance. The observed shift could result both from a greater extension of the giant branch to low temperature and from higher metal abundance resulting in stronger TiO bands (the feature which has the largest effect on $\left(V-I_{K}\right)$ ) at given Te. We note, however, that the metal poor cluster $\omega$ Cen has, in addition to a group of variables with $\left(V-I_{K}\right)$ $=1.4$, two (V6 and V17) with $\left(V-I_{K}\right) \sim 2.4$ (Dickens et al., 1972). The situation may thus be more complex, though the finer details are only apparent in such rich clusters as $\omega$ Cen and 47 Tuc. 


\section{Mira Variables}

Mira variables are readily detectable by their large amplitude and very red $\left(V-I_{K}\right)$ when faint. Several were recognised as such for the first time during this investigation. (Table I). The period of V10 in NGC 6637 has been found from Cape and Radcliffe plates to be about 195 days, similar to that of V4. V4 in NGC 6553 may have $P=270$ days, though periods near 150 and 210 days are perhaps not excluded. Osborne's (1968) variable, V1, in NGC 5927 has only twice been seen bright on Radcliffe plates, at $V \sim 15$, on 1 August 1954 and on 1 April 1968. Negative observations, the variable being fainter than $V \sim 18$ on 4 occasions and fainter than $V \sim 16.5$ on 6 occasions, argue strongly against a period near 200 days. $P=300-330$ days would satisfy all the negative observations.

These long periods would normally be accompanied by a fainter $M_{V}(\max )$ than that for the 200 day variables, in line with the gentler slope of the giant branch in the clusters of group C. We might regard V42 $\left(P=149^{\mathrm{d}}\right)$ in $\omega$ Cen as a Mira variable on the strength of the spectroscopic results of Dickens et al. (1972), in which case there would be a trend of increasing period with increasing metal abundance, from $150^{d}$ in $\omega$ Cen to $\sim 200^{\mathrm{d}}$ in NGC 6712,6637 and 104, and to $\sim 300^{\mathrm{d}}$ in NGC 6553 and NGC 5927. Confirmation of this result is highly desirable and determination of definitive periods for the eight outstanding cases (V1, V3, (V4) in NGC 6356; V1, V2, V4 in NGC 6388; $\mathrm{V} 1$ in NGC 5927; V4 in NGC 6553) would be most valuable. These variables are almost all severely crowded or located in regions of high surface density of faint stars, so that a moderately large telescope will be needed, preferably using infrared plates to suppress the bluer stars in the clusters.

\section{Acknowledgements}

We are indebted to Dr A. D. Thackeray for the loan of his plates and finding chart for NGC 6553: TLE is indebted to Dr M. W. Feast for many valuable discussions; to Mr G. A. Harding for the use of plates of NGC 6637 taken at the Royal Observatory, Cape; and to Professor D. W. N. Stibbs for his hospitality at the University Observatory, St. Andrews, where most of the blink microscope work was done. J. W. M. wishes to acknowledge the support of the Radcliffe-Henry Skynner Fellowship and of the Science Research Council during this project.

\section{References}

Arp, H., Brueckel, F., and Lourens, J. V. B.: 1963, Astrophys. J. 137, 228.

Bergh, S. van den: 1967, Astron. J. 72, 70.

Brooke, A. L.: 1969, Thesis, Australian National University.

Catchpole, R. M., Feast, M. W., and Menzies, J. W.: 1970, Observatory 90, 63.

Dickens, R. J., Feast, M. W., and Lloyd Evans, T.: 1972, Monthly Notices Roy. Astron. Soc., 159, 337.

Eggen, O. J.: 1972, Astrophys. J. 172, 639.

Feast, M. W.: 1972, Quart. J. Roy. Astron. Soc. 13, 191.

Feast, M. W. and Thackeray, A. D.: 1960, Monthly Notices Roy. Astron. Soc. $120,463$. 
Fourcade, C. R. and Laborde, J. R.: 1966, Atlas y Catálogo de Estrellas Variables en Cúmulos Globulares al sur de $-29^{\circ}$, Cordoba.

Hartwick, F. D. A. and Hesser, J. E.: 1972, Astrophys. J. 175, 77.

Hartwick, F. D. A. and Sandage, A. R.: 1968, Astrophys. J. 153, 715.

Hogg, H. S.: 1955, Publ. David Dunlap Obs. 2, 35.

Kron, G. E. and Smith, J. L.: 1951, Astrophys. J. 113, 324.

Lloyd Evans, T. and Menzies, J. W.: 1971, Observatory 91, 35.

Osborne, W.: 1968, Observatory 88, 26.

Rosino, L.: 1962, Mem. Soc. Astr. Ital. 33, 351.

Sandage, A. R. and Katem, B.: 1964, Astrophys. J. 139, 1088.

Sandage, A. and Smith, L. L.: 1966, Astrophys. J. 144, 894.

Sandage, A. R. and Wallerstein, G.: 1960, Astrophys. J. 131, 598.

Wildey, R. L.: 1961, Astrophys. J. 133, 430.

\section{DISCUSSION}

Hogg: How many of these variables are new? Lloyd-Evans: About 50. 